DOI: 10.20472/IAC.2017.029.028

\title{
ELENA RAEVSCHI
}

Nicolae Testemitanu State University of Medicine and Pharmacy of the Republic of Moldova, Moldova, Republic of

\section{BEHAVIORAL RISK FACTOR SURVEILLANCE SYSTEM DEVELOPMENT IN THE REPUBLIC OF MOLDOVA}

\begin{abstract}
:
In the Republic of Moldova cardiovascular diseases are considered to be one of the most important public health issues, showing about $56 \%$ of the total mortality structure in the last decade. According to the World Health Organization behavior risk factor is considered as main target of intervention in prevention and control of noncommunicable diseases, including cardiovascular diseases. Despite this fact, an ongoing monitoring of behavioral risk factor is not implemented in the health information system in the Republic of Moldova.

The aim of the study was to evaluate the feasibility of a new implementation of the ongoing behavioral risk factor surveillance system in the Republic of Moldova.

There was performed many approaches as: SWOT analyses and organizational experiment which results suggested a Delphi survey initiation. There was applied the research design of transversal study using the methodology based on the U.S. behavioral risk surveillance system standards. The systematic random sampling was performed in order to select 800 land telephone numbers. The adults aged 18-69 years were considered eligible for land phone interview provided by the trained staff.

As a result of the analysis of data, it has been found that interviewers reached the respondents in $37.5 \%(95 \% \mathrm{Cl}, 34.21 \%-40.91 \%)$ of cases.

In conclusion, the low resolution rate is determined by the high level of unresolved cases. The ways to decrease the number of unknown eligibility category is to apply good practices of developed proven ongoing behavioral risk factor surveillance systems of such countries as the U.S.A., Italy and Canada by performing Delphi survey. The consensus on the opinions of experts will contribute to provide more credible evidence based recommendations for a new implementation of the behavioral risk factor surveillance system in the Republic of Moldova.
\end{abstract}

\section{Keywords:}

behavioral risk factor surveillance system

JEL Classification: $|18| 12,, \mid 10$ 


\section{Introduction}

Cardiovascular diseases are placed first for all death causes of the population over the last 10 years in the Republic of Moldova. The current health care system in the Republic of Moldova is not adequately focused on surveillance of behavioral risk factors: there is no system for systematically collecting and assessment of behavioral risk factor data.

There was performed many approaches, in order to contribute of the ongoing behavioral risk factor surveillance system (BRFSS) development and to evaluate the feasibility of a new implementation in the Republic of Moldova, such as:

1. SWOT analyses of developed behavioral risk factor surveillance systems of the USA, Canada and Italy: the common strengths, weaknesses, opportunities and threats of the existing systems were evaluated (Pautz Linda et al., 2016);

2. Organizational experiment: there was applied the research design of transversal study mainly using the methodology based on the U.S. behavioral risk surveillance system standards in the conditions of the Republic of Moldova where was determined challenges of the surveillance quality (Ababii lon et al, 2016).

In this way, the systematic random sampling was performed in order to select 800 land telephone numbers. The adults aged 18-69 years were considered eligible for land phone interview provided by the trained staff.

According to the eligibility study participants were structured as follows: known eligibility in $33.25 \%(95 \% \mathrm{Cl}, 30.07 \%-36.59 \%)$; unknown eligibility (unresolved cases) in $62.5 \%(95 \% \mathrm{Cl}, 59.09 \%-65.79 \%)$ and not eligible in $4.25 \%(95 \% \mathrm{Cl}, 3.06 \%-5.88 \%)$ of total sample cases.

A completed interview was given by $93.2 \%(95 \% \mathrm{Cl}, 89.56 \%-95.68 \%)$ of eligible participants and $1.9 \%(95 \% \mathrm{Cl}, 0.81 \%-4.32 \%)$ of them gave partially completed interview. With that, eligible respondent refusal was found in only $4.9 \%(95 \% \mathrm{Cl}$, $2.88 \%-8.18 \%$ ).

Unknown eligibility was categorized in $44.4 \%(95 \% \mathrm{Cl}, 40.1 \%-48.78 \%)$ as no answer calls despite repeated attempts, $36 \%(95 \% \mathrm{Cl}, 31.91 \%-40.3 \%)$ - answering device, unknown whether eligible, $19.2 \%(95 \% \mathrm{Cl}, 15.99 \%-22.88 \%)$ - telecommunication barrier and in $0.4 \%(95 \% \mathrm{Cl}, 0.1 \%-1.4 \%)$ - physical impairment.

Not eligible category of persons was defined in $97.06 \%(95 \% \mathrm{Cl}, 85.09 \%-99.48 \%)$ as out of sample and in $2.94 \%(95 \% \mathrm{Cl}, 0.52 \%-14.91 \%)$ as non-residence.

As a result of the analysis of data, it has been found that interviewers reached the respondents in $37.5 \%(95 \% \mathrm{Cl}, 34.21 \%-40.91 \%)$ of cases: the low resolution rate is determined by the high level of unresolved cases. In this context the study results suggest that the ways to decrease the number of unknown eligibility category is to apply good practices of developed proven ongoing behavioral risk factor surveillance systems of such countries as the U.S.A., Italy and Canada by performing Delphi survey. 


\section{Delphi method}

For the first time in the late 1950s, in a research by U.S. RAND Corporation the Delphi technique was introduced for the scientific study of experts' opinion on military defense field. However, for the security reason the methodology was not widely used over ten years and in 1963 Dalkey and Halmer introduced it for the first non-military use. This method became highly popular in the academic studies from the mid-90s. (Arash Habibi et al., 2014). In the literature, Delphi has been applied in various fields such as program planning, needs assessment, policy determination, and resources utilization (Hsu C. \& Sandford B., 2007). Currently, the method is becoming increasingly popular in health and social research.

The first step for the application of the Delphi technique is to determine the problem and then select experts (Delphi panel) in the area of concern. The questionnaire is considered as a tool of method that is distributed to the panel members. After collecting the data the analyses for consensus in response is performed. The next questionnaire is developed only on base of non-consensus questions and fed back to the panel. This process is repeated until consensus is reached (usually two rounds). A final report is developed based on this process.

\section{The Delphi method of the Behavioral Risk Factors Surveillance System (BRFSS) Development in the Republic of Moldova}

The point of the Delphi study is to gather expert opinion on different policy issues concerning the management of a risk factors surveillance system. These expert opinions and arguments will, along with research findings of other work sets of the Control and Prevention of non-communicable diseases, be part of the foundation for the development of Behavioral Risk Factors Surveillance System (BRFSS) in the Republic of Moldova.

The Delphi study includes two written Delphi rounds to complete a questionnaire (via e-mail). The Delphi panel includes experts from countries with expertise in the field of Behavioral Risk Factors Surveillance: U.S.A., Italy, Canada, France, Romania and Republic of Moldova.

The main objective of the Development of BRFSS in the Republic of Moldova project is to provide evidence of good practices based on a model of implementation of Behavioral Risk Factors Surveillance System which is adjusted to the local needs in order to support the National Strategy for Prevention and Control of NonCommunicable Diseases 2012-2020.

The specific aim of the Development of Behavioral Risk Factors Surveillance System (BRFSS) in the Republic of Moldova Delphi study is to gather expert opinion on a variety of national and international health policies regarding the management of surveillance related risk factors. 
For the study it was determined to have experts in the field of management of risk factors surveillance from the countries which implemented the ongoing BRFSS using the U.S standards. A total of 40 of risk factors surveillance experts' management will be invited to participate from USA, Europe, and Canada.

The first Delphi round questionnaire was prepared based on several sources: Action plan of National Strategy for Prevention and Control of Non-Communicable Diseases in the Republic of Moldova 2012-2020, problem areas in the international and national control and prevention of risk factors as found in the literature review, integrated SWOT analyses, and research results from pilot cross-sectional telephone survey test conducted in the Republic of Moldova $(n=800)$ mainly based on the US BRFSS's protocol (CDC, 2013).

The first Delphi round questionnaire was structured around the policy issues of management of the Behavioral Risk Factors Surveillance System adjusted to the adapted checklist for Evaluating Public Health Surveillance Systems (CDC, 2001) listed below:

1. Importance of behavioral risk factors surveillance system development

2. Impact on health system of the ongoing BRFSS

3. Roles and responsibilities of central and regional levels

4. Operation of the surveillance system: purpose

5. Operation of the surveillance system: components of system

6. Resources used to operate the surveillance system

7. System attribute: gather credible evidence regarding the performance of the surveillance system

The First Round Delphi questionnaire is consisted of a total of 61 questions. A 9-point Likert agreement scale was applied to measure the strength of a subject's agreement with a clear statement (1- completely disagree and 9- completely agree).

All questionnaires will be provided with comments and suggestions boxes after each statement that will be used along with quantitative analyses. The comments and suggestions would help to develop priorities among existing ongoing BRFSS performance or propose other solutions that would be more appropriate to specified conditions and expectations for a new implementation.

In order to improve the quality of questionnaire will be performed the pilot round: sending the first round questionnaire to the three experts who do not belong to the approved Delphi panel list.

Items included in the First Round Delphi questionnaire were as following: 


\section{General}

\section{Importance of behavioral risk factors surveillance system development}

1. Ongoing surveillance of modifiable (behavioral and biological) risk factors is necessary along with mortality and morbidity vital statistics monitoring

2. Prevalence surveys of behavioral risk factors conducted with long-term periodicity by international organizations exclude the needs to develop an ongoing surveillance system of behavioral risk factors local-based random telephone survey in the country.

3. Development of ongoing behavioral risk factors surveillance system (BRFSS) based on international good practices contribute to global data comparison.

\section{Impact on health system of the ongoing BRFSS}

4. Cross-sectional pilot survey at the regional level is required before the future implementation at the national level

5. The results of ongoing BRFSS are used to support program planning and evaluation, public policy development and improve community awareness regarding the risks for chronic diseases

6. The results of ongoing BRFSS assure continuum updating of data set providing permanently actual evidence

7. Regarding the public health impact evaluation, ongoing surveillance of modifiable risk factors is more logical and efficient to be performed along with monitoring of premature deaths than classical approach of mortality monitoring.

8. Ongoing BRFSS as a tools' collection covers population regardless of their addressability in the medical institution: provided interview is an opportunity for health promotion as well.

9. Since there has been the implementation of an ongoing surveillance system of risk factors in your country, it has produced positive trends for premature cardiovascular mortality

10. Please list up to 5 changes in control and prevention of cardiovascular diseases relevant to your country since an ongoing surveillance system of risk factors was implemented:

\section{Roles and responsibilities of central, regional and local levels}

11. Engage the stakeholders at three distinct levels: national, regional and local.

12. Local Health Units (LHU) having access to all residents' demographic data must be the first-line ongoing telephone survey that collects information for the surveillance system of public health (National surveillance system with local implementation). 


\section{Operation of the surveillance system: purpose}

\section{Health -related risk factor events under surveillance}

In terms of providing a complex surveillance of risk factors, the system is required to take into account the following events:

13. Behavioral risk factors: smoking status, healthy diet, alcohol consumption, and physical activity.

14. Biological risk factors: blood pressure, total serum cholesterol, fasting blood glucose, and BMI.

15. Ongoing surveillance of modifiable risk factors is required along with monitoring of individual coexisting morbidities.

\section{Surveillance indicators}

Calculation of core monitoring indicators:

16. Events related to monitoring indicators.

17. Quality related indicators of system performance (Response rates).

18. Indicators must respect international common standards.

\section{Level of integration}

In terms of providing an integrated surveillance of risk factors, the system is required to take into account the following:

19. Using the standards of existing ongoing BRFSS which permits comparability among surveys at the international level.

20. Ongoing surveillance of modifiable risk factors is required along with monitoring of mortality (inclusively premature deaths) and morbidity, all being integrated as an informational system.

21. Systematic and on-going approaches in the surveillance of risk factors help to provide useful information for the continual monitoring of public health trends.

\section{Operation of the surveillance system: components of system}

\section{Population under surveillance}

In terms of premature deaths, assessment is more logical and efficient:

22. The study population should consist of persons aged between 18 and 69 old rather than the study population consisting of persons aged 18+ years old.

In terms of mitigating falling survey resolution and response rate, it is more logical and efficient to:

23. Use an approach to select respondents enrolled in the lists of local health units vital statistics, updated on 1st January of the year of the survey than to use the Random Digit Dialing (RDD) approach to select the phone numbers by randomly selecting from commercially available lists of telephone numbers. 


\section{Period of time data collection}

In order to mitigate falling survey response rates, it is more logical and efficient to do a:

24. Sampling based on a monthly collection and reporting frame which may not provide enough time to contact respondents and for follow-up refusal conversations.

25. Sampling based on a monthly collection switched to a four month reporting frame which may provide more time to contact respondents for a refusal conversation.

\section{Data collection}

In order to mitigate falling survey response rates, it is more logical and efficient to do a:

25. To provide telephone interview data collection by a Local Health Unit (LHU) working team comprised of coordinators (family doctor, statistician), interviewers (nurses), and public health professionals.

26. To provide telephone interview data collection by a Local Public Health Unit (LPHU) working ream comprised of several public health professionals and statisticians.

27. For each unit the monthly, and respectively, annual minimum sample size of completed interview is defined at the beginning of year.

\section{Reporting source of data, analyses and dissemination}

28. Entry and reporting of data is managed monthly at the local level and at the end of calendar year data are edited at the central level.

29. Data reported by collecting units every four months, respecting monthly entry of minimum sample size (three data collections cycles): at the end of calendar year data are edited at the central level.

\section{Data management}

30. National coordinating group supervises the application of methods and standards of the process

31. National technical group supervises process of verification and correction of the interviews and makes available dataset after prior quality controls are applied to programs.

32. Regional coordinating group gives assistance and support to the local working teams.

\section{Resources used to operate the surveillance system}

\section{Funding sources}

33. New implementation of BRFSS using existing Health System organization and management provide affordable expenditures.

34. Please list the ways you find relevant to providing a new implementation of ongoing BRFSS local-based random telephone surveys that have minimal expenditures: 
35. Please list up to five sectors you find are relevant to be involved in the financial maintenance of essential services of an ongoing BRFSS:

\section{Personnel requirements}

36. Telephone survey is conducted by trained staff at the local level.

37. Technical and methodological assistance for training is provided by a coordinating group at the National level.

38. Local Working group is composed of coordinators, interviewers, and public health experts.

39. Coordinators are a medical doctor and a statistician already working in LHU.

40. Interviewers are nurses already working in LHU.

\section{System attribute: gather credible evidence regarding the performance of the surveillance system}

\section{Simplicity}

41. Flow chart is structured according to three distinct levels: national, regional and local.

42. Determine standards for system operation and assessing the performance.

\section{Flexibility}

43. Along with a fixed core component of the questionnaire, it is required to provide rotating core components (sets of questions, asked in alternating years by all participating LHUs that address different topics).

44. Along with fixed core components of the questionnaire, it is required to provide optional modules (questions that Regions select in their questionnaires to achieve needed information).

45. Along with fixed core component of the questionnaire, it is required to provide emerging modules (a few questions for brief periods of time).

46. Only Fixed core components of questionnaire are required to be provided during the implementation period (sets of standardized questions, asked in deferent periods of time by all participating LHUs).

\section{Data quality}

47. To utilize the Nationwide standardized structured questionnaire and the surveillance indicators calculation.

48. To share a common information system and database.

49. To provide central supervision and support for training, data analyses and communications activities. 


\section{Acceptability}

50. Extracting the sample from LHUs lists of residents vs. extracting the sample from random digit dialing will improve respondent's accessibility

51. Data collection provided by Local Health Units vs. Data collection provided by Public health Units will improve respondent's cooperation

52. Please indicate number of questions to include in the questionnaire that you consider acceptable by respondents in order to limited refusal rate

53. Please indicate interview time you consider acceptable by respondents in order to limit refusal rate

\section{Sensitivity}

54. Health promotion interventions improve the ability of persons to understand the questions and correctly identify their status.

55. Continued information about the surveillance system provided not only on regular medical check-up visits but also through the media will improve the willingness of respondents to report their status.

\section{Representativeness}

In terms of premature deaths assessment in association with prevalence of modifiable risk factors, it is more logical and efficient to have a:

56. Stratified sampling by sex and age 18-69 (18-34, 35-49, 50-69 years).

57. Stratified sampling by sex and age 18+ (18-44, 45-64, 65+ years).

\section{Timelines}

Regarding the timelines friendly system it is more logical and efficient to have:

58. Electronic data collection from reporting sources including entry data by webbased system (local/regional/national access).

59. Use of electronic data from local level entry by Microsoft Excel. All completed entries are gathered and processed on a regional level.

60. Use of paper based entries from Local levels that are not IT equipped. All completed entries are gathered and processed on a regional level.

\section{Stability}

61. Please list up to three conditions you find are relevant to maintain the stability of ongoing BRFSS:

In order to avoid information bias the first round questionnaire will be provided along with instruction.

The analyses of the replies will be done by looking at the statistics using SPSS and qualitative data provided by the respondents in all Delphi rounds. 


\section{Conclusion}

The results of Delphi survey will contribute to provide more credible evidence-based recommendations for a new implementation of the behavioral risk factor surveillance system in the Republic of Moldova.

\section{Reference}

ARASH HABIBI, AZAM SARAFRAZI, SEDIGHEH IZADYAR (2014). Delphi Technique Theoretical Framework in Qualitative Research. International Journal of Engineering and Sciences. 2014, Vol.3, Issue 4, p.8-13.

DALKEY N.C., HELMER O.(1963). An Experimental Application of the Delphi method to the use of experts. Management Science. 1963, 9(3), p.458-467.

HSU C., \& SANDFORD B. (2007). The Delphi Technique: Making Sense Of Consensus. Practical Assessment. 2007, Research \& Evaluation, Volume 12, Nr.10, p. 1-8.

LINDA PAUTZ, ELENA RAEVSCHI, ASHA PATEL, EUGENIA CIUBOTARU (2016). Development of Behavioral Risk Factor Surveillance System Management in the Republic of Moldova Based on the U.S. BRFSS Standards. International Journal of Business and Management Invention. 2016, Vol.5, Issue 7, p.33-36.

ABABII ION, ELEONORA VATAMAN, ELENA RAEVSCHI, LINDA PAUTZ (2016). Pilot CrossSectional Telephone Survey Test mainly based on the U.S. BRFSS's Protocol Conducted in the Republic of Moldova: Challenges of the Surveillance Quality. International Journal of Humanities and Social Sciences. 2016, Vol.5, Issue 6, p.73-80.

CDC (2001). Updating Guidelines for Evaluating Public Health Surveillance Systems. Centers for Disease Control and Prevention. 2001, 50(RR13); 1-35. Retrieved from: http://www.cdc.gov/mmwr/preview/mmwrhtml/rr5013a1.htm\#fig1.

CDC (2013). Behavioral Risk Factor Surveillance System Data User Guide. Centers for Disease Control and Prevention. $2016 . \quad$ Retrieved https://www.cdc.gov/brfss/data documentation/pdf/userguidejune2013.pdf

CDC (2015). Behavioral Risk Factor Surveillance System Questionnaire 2016. Centers for Disease Control and Prevention. 2016. Retrieved from: https://www.cdc.gov/brfss/questionnaires/pdfques/2016 brfss questionnaire final.pdf

National Strategy for Prevention and Control of Noncommunicable Diseases 2012-2020, June 22, 2012, Monitorul Oficial Nr.126-129, Art. Nr:412. Retrieved from: http://lex.justice.md/index.php?action=view\&view=doc\&lang=1\&id=343682. 